\title{
A case report of COVID-19 with false negative RT-PCR test: necessity of chest CT
}

\author{
Hao Feng ${ }^{1,2} \cdot$ Yujian Liu ${ }^{1,2} \cdot$ Minli Lv ${ }^{1,2}$ Jianquan Zhong ${ }^{1,2}$ \\ Received: 23 February 2020 / Accepted: 28 March 2020 / Published online: 7 April 2020 \\ (c) Japan Radiological Society 2020
}

\begin{abstract}
The definite diagnosis of corona virus disease 2019 (COVID-19) is based on the viral isolation or positive result of polymerase chain reaction (PCR) from sputum, or nasal swab, or throat swab. However, the sensitivity to detect COVID-19 of real time (RT)-PCR is reported to be lower than that of chest CT. We report a case of 34-year-old man who was diagnosed as negative for COVID-19 based on the four sequential RT-PCR tests of his pharyngeal swab. Chest CT showed patchy groundglass opacity on admission, and it rapidly progressed to segmental mixed consolidation and ground-glass opacity 3 days after admission, and it resolved in left upper lobe, but showed multifocal ground-glass opacities 7 days after admission, and they resolved within 2 weeks. The fifth RT-PCR test finally revealed positive results at the fifth day after admission. It is difficult to distinguish COVID-19 pneumonia from other viral pneumonia on CT findings alone; however, we emphasize the utility of chest CT to detect early change of COVID-19 in cases which RT-PCR tests show negative results.
\end{abstract}

Keywords COVID-19, 2019 corona virus disease $\cdot$ RT-PCR, real-time fluorescence polymerase chain reaction $\cdot$ CT, computed tomography $\cdot$ CXR, chest radiography

\section{Case report}

Since December 2019, a cluster of pneumonia cases of unknown etiology has been announced in Wuhan, China, the pathogen of which is a novel coronavirus [1]. It broke out across China in just a week and spread rapidly around the world in a month [2]. WHO has officially named the disease with 2019 Coronavirus disease as COVID-19 [3, 4].

A 34-year-old man presented to the community hospital with 1-day history of chill and fever $\left(38.7{ }^{\circ} \mathrm{C}\right)$ of unknown cause. Five days ago, he just passed Wuhan high-speed rail station in China on train. Coarse breath sounds of both lungs were heard at auscultation. Laboratory studies showed white blood cell count $5,660,000 / \mathrm{mL}$, lymphocyte cell count $1,150,000 / \mathrm{mL}$, the white blood cell differential count showed 70.6\% neutrophils (normal range 40-75\%), 0.0\% eosinophils (normal range $0.4-8 \%$ ), and $8.5 \%$ monocytes

Jianquan Zhong

zhongjianquan.2010@qq.com

1 Department of Radiology, Zigong First People's Hospital, Zigong 643000, People's Republic of China

2 Zigong Infectious Disease Hospital, Zigong 643000, People's Republic of China (normal range 3-10\%). There were elevated blood levels for hypersensitivity C-reactive protein $(23.6 \mathrm{mg} / \mathrm{L}$; normal range $0-5 \mathrm{mg} / \mathrm{L}$ ), Prothrombin time $(13.5 \mathrm{~s}$; normal range 9.0-16.0 s), Activated partial thromboplastin time (40.3 s; normal range $27.0-45.0 \mathrm{~s}$ ).

But real-time fluorescence polymerase chain reaction (RT-PCR) of the patient's pharyngeal swab for severe acute respiratory syndrome-Corona virus-2 (SARS-CoV-2) nucleic acid was always negative for four times at disease onset. The clinical doctor could not diagnose as COVID-19 for the negative RT-PCR testing in its early stage.

CXR at disease onset (Fig. 1a, red square) showed patchy opacities in the left middle lung field. Thereafter, he was transferred immediately to the superior hospital, and chest CT showed patchy ground-glass opacity in the upper lobe of the left lung on admission (Fig. 1b), and it rapidly progressed to segmental mixed consolidation and ground glass opacification with air-bronchogram 3 days after admission (Fig. 1c), and it resolved in left upper lobe and also showed multifocal ground-glass opacities 7 days after admission (Fig. 1d1, axial image; Fig. 1d2, ray-summation image; Fig. 1d3, pseudo color MIP; Fig. 1d4, coronal image), and they resolved within 2 weeks (Fig. 1e, coronal image). Fortunately, this patient was diagnosed as "presumed cases" based 


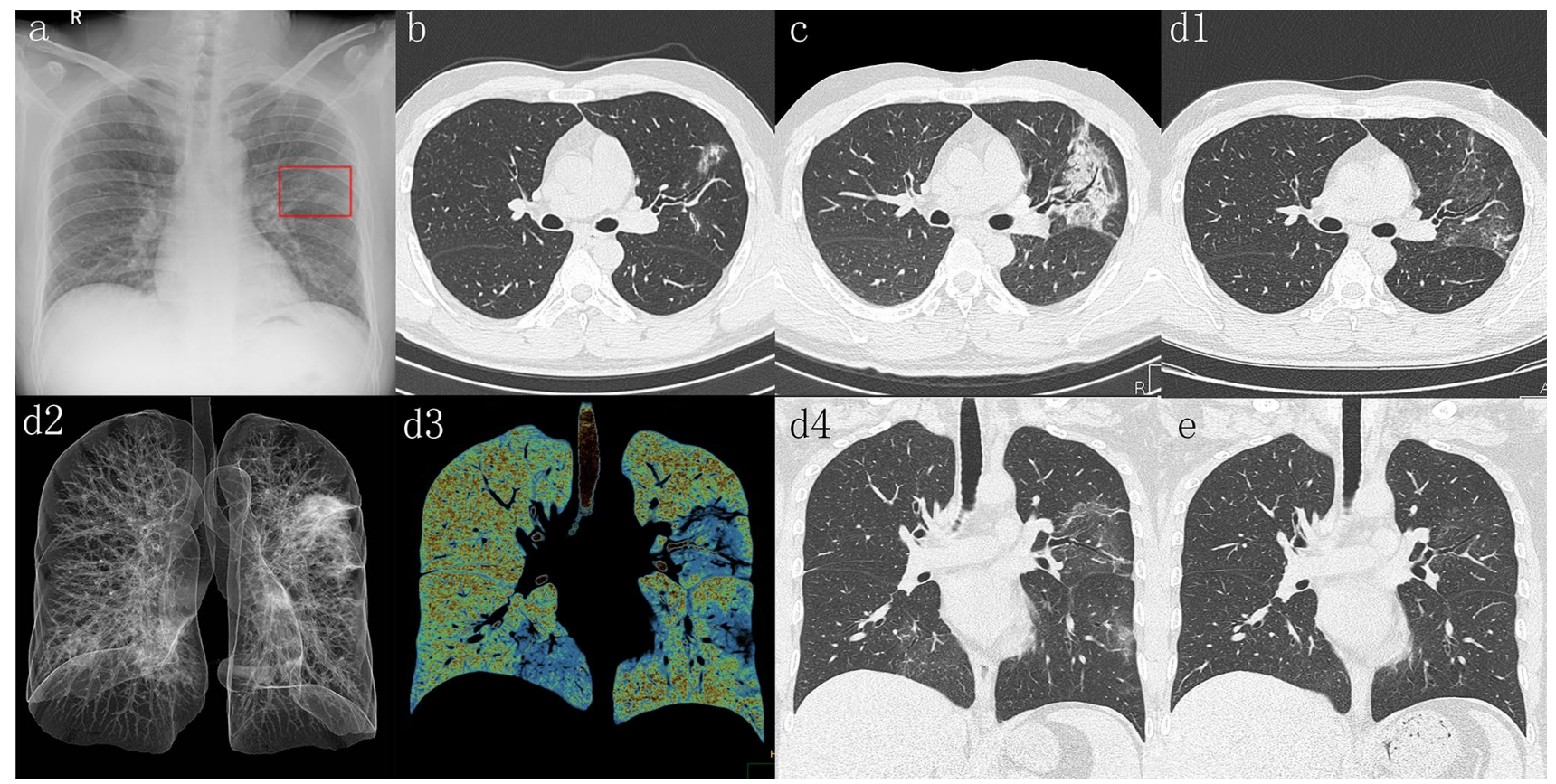

Fig. 1 a CXR shows infiltrate in the left middle lung field (red square). b High resolution CT with $1 \mathrm{~mm}$ thickness after admission at disease onset shows patchy ground-glass opacity in left upper lobe. c Follow-up CT 3 days after admission shows evolution to a segmental mixed pattern of ground-glass opacities and consolidation that grow larger with air bronchogram in left upper lobe. d Follow-

on early CT findings, the patient was immediately isolated for clinical monitoring so as not to cause regional spread. The fifth RT-PCR test was positive on the fifth day after admission; this patient was finally confirmed as COVID-19. Serial imaging studies in the Fig. 1d, e illustrated that the patient recovered after treatment.

On the basis of epidemiologic characteristics, clinical manifestations, chest CT images, and laboratory findings, the diagnosis of COVID-19 pneumonia was made. When the RT-PCR test for swab was negative at early stage, the chest image would play an important role in diagnosis [5-7]. Therefore, we need to strengthen the recognition of image changes to help clinicians to diagnose quickly and accurately. It is difficult to distinguish COVID-19 pneumonia from other viral pneumonia on CT findings alone; however, high-resolution CT (HRCT) of the chest is critical for early detection and improvement of diagnostic confidence for patient with COVID-19 amid possible negative RT-PCR period.

\section{Compliance with ethical standards}

Conflict of interest The authors declare that they have no conflict of interest. up CT 7 days after admission (d1, axial image; d2, ray-summation image; d3, pseudo color MIP; d4, coronal image) shows multifocal bilateral ground-glass opacities and improvement of mixed groundglass opacities and consolidation in left upper lobe. e Coronal MPR image obtained 2 weeks after admission shows marked improvement of multifocal ground-glass opacities in both lungs

Research involving human and/or animal participants This article does not contain any studies with human participants or animals performed by any of the authors.

\section{References}

1. Zhu N, Zhang D, Wang W, et al. A novel coronavirus from patients with pneumonia in China, 2019. N Engl J Med. 2020. https://doi. org/10.1056/NEJMoa2001017.

2. Velavan TP, Meyer CG. The Covid-19 epidemic. Trop Med Int Health. 2020. https://doi.org/10.1111/tmi.13383.

3. World Health Organization. Coronavirus disease 2019. https://www. who.int/emergencies/diseases/novel-coronavirus-2019.

4. Jiang S, Shi Z, Shu Y, et al. A distinct name is needed for the new coronavirus. Lancet. 2020. https://doi.org/10.1016/S0140 -6736(20)30419-0.

5. Kanne JP. Chest CT findings in 2019 novel coronavirus (2019$\mathrm{nCoV}$ ) infections from Wuhan, China: key points for the radiologist. Radiology. 2020;10:11. https://doi.org/10.1148/radiol.2020200241.

6. Xie X, Zhong Z, Zhao W, et al. Chest CT for typical 2019-nCoV pneumonia: relationship to negative RT-PCR testing. Radiology. 2020. https://doi.org/10.1148/radiol.2020200343.

7. Ai T, Yang Z, Hou H, et al. Correlation of chest CT and RT-PCR testing in coronavirus disease 2019 (COVID-19) in China: a report of 1014 cases. Radiology. 2020. https://doi.org/10.1148/radio 1.2020200642 .

Publisher's Note Springer Nature remains neutral with regard to jurisdictional claims in published maps and institutional affiliations. 